\title{
Effet des Perturbations Électriques sur les Appareils de Radiologie À Rayons X À Haute Fréquence (HF) Alimentés par le Réseau Électrique Camerounais: Cas des Coupures D’Énergie
}

\author{
Kpalang Docta Basile \\ Doctorant, Université de Ngaoundéré, Cameroun \\ CURES, Université de Yaoundé I, Cameroun \\ Onanena Raïssa \\ PhD, CURES, Université de Yaoundé I, Cameroun \\ Ntsama Eloundou Pascal \\ Maitre de Conférences, Université de Ngaoundéré, Cameroun
}

Ndarwe Djakba

$\mathrm{PhD}$, Université de Douala, Cameroun

Ele Pierre

Professeur des Universités, CURES, Université de Yaoundé I, Cameroun

Njaka Manyol Eric Martial

Expert, Agence National de Radioprotection, Cameroun

Nana Nwedjiwe Narcisse

Ingénieur Biomédical, Hôpital Général de Douala, Cameroun

Doi:10.19044/esj.2021.v17n37p156

Submitted: 02 March 2021

Accepted: 04 October 2021

Published: 31 October 2021
Copyright 2021 Author(s)

Under Creative Commons BY-NC-ND 4.0 OPEN ACCESS

Cite As:

Kpalang D.B., Onanena R., Ntsama Eloundou P., Ndarwe Djakba, Ele P., Njaka Manyol E.M. \& Nana Nwedjiwe N. (2021). Effet des Perturbations Électriques sur les Appareils de Radiologie À Rayons X À Haute Fréquence (HF) Alimentés par le Réseau Électrique Camerounais: Cas des Coupures D’Énergie. European Scientific Journal, ESJ, 17(37), 156. https://doi.org/10.19044/esj.2021.v17n37p156

\section{Résumé}

L’organisation Mondiale de la Santé estime que plus d'un tiers des pannes des appareils médicaux utilisés dans les pays du Sud sont causées par un réseau défaillant. Le présent article porte sur l'effet des coupures d'énergie électrique sur les appareils de radiologie à rayons $\mathrm{X}$ à haute fréquence, alimentés par le réseau électrique Camerounais. Des mesures des grandeurs électriques aux bornes de onze appareils de radiologie ont été effectuées à 
l'aide d'un analyseur de réseau et d'un $\mathrm{kVp}$ mètre. Le logiciel d'analyse de données Win-PQ a permis d'analyser les données enrégistrées. Les résultats montrent que, des coupures ayant eu lieu pendant la production des rayonnements $\mathrm{X}$, engendrent des surtensions, ce qui augmente la quantité de rayonnement irradié au patient avec risques de blessures et détérioration d'appareillage. Ces surtensions sont d'amplitudes plus élevées lorsque les appareils fonctionnent en hyper résonance. Ceci souligne la nécessité de revoir les normes de conception et de fabrication des appareils de radiologie utilisés dans les pays du Sud dont le réseau électrique est soumis en permanence à des coupures. L'une des solutions envisageable serait de recourir aux sources d’énergies renouvelables hors réseau.

Mots clés: Radiologie, Rayons X, Coupures D’énergie Electrique, Surtension, Normes

\title{
Effect of Electrical Disturbances on High Frequency X-Ray Radiology (HF) Equipment Supplied by The Cameroonian Electricity Grid: Case of Power Cuts
}

\author{
Kpalang Docta Basile \\ PhD student, University of Ngaoundere, Cameroon \\ CURES, University of Yaounde I, Cameroon \\ Onanena Raïssa \\ $\mathrm{PhD}$, CURES, University of Yaoundé I, Cameroon \\ Ntsama Eloundou Pascal \\ Lecturer, University of Ngaoundere Cameroon \\ Ndarwe Djakba \\ PhD, University of Douala, Cameroon \\ Ele Pierre \\ University Professor, CURES, University of Yaounde I, Cameroon \\ Njaka Manyol Eric Martial \\ Expert, National Agency for Radiation Protection, Cameroon \\ Nana Nwedjiwe Narcisse \\ Biomedical Engineer, Douala General Hospital, Cameroon
}

\begin{abstract}
The World Health Organization estimates that more than one third of the breakdowns of medical equipment used in the countries of the South are caused by a faulty network. This paper investigates the effect of power outages on high-frequency X-ray machines powered by the Cameroonian electrical grid. Measurements of electrical quantities at the terminals of eleven X-ray
\end{abstract}


machines were made using a network analyzer and a $\mathrm{kVp}$ meter. Win-PQ data analysis software was used to analyze the recorded data. The results show that power cuts during the production of $\mathrm{X}$-rays cause overvoltages, which increase the intensity of radiation delivered to the patient, and also the risk of injury and damage to the equipment. These surges are of higher amplitudes when the equipment is operating in hyper-resonance. This underlines the need to review the design and manufacturing standards of X-ray equipment used in countries in the South where the electrical network is permanently subject to power cuts. One of the solutions recommended would be the use of renewable energy sources off-grid system.

Keywords: Radiology, X-Rays, Power Outages, Surge, Standards

\section{Introduction}

L’Organisation Mondiale de la Santé (OMS) accorde aux dispositifs médicaux le rôle d'outils indispensables à la fourniture de soins de santé et à l'amélioration de la santé des populations. En 2007, l'OMS a mis en place le projet Dispositifs Médicaux Prioritaires (DMP) pour déterminer si les dispositifs médicaux actuellement sur le marché mondial répondaient aux besoins des prestataires de soins de santé et des patients dans le monde entier. Ce projet visait à identifier les lacunes sur le marché des dispositifs médicaux et les obstacles potentiels à l'utilisation optimale des dispositifs médicaux en tant qu'outils de santé publique et à proposer des mesures correctives basées sur des données objectives (Organisation Mondiale dela Santé, 2012).

L'OMS estime que les appareils électriques des hôpitaux des pays du Sud proviennent à 95\% de l'Europe (MAINSAH, 2008) (MALKIN, 2007). Ils sont par conséquent conçus sur la base des standards Européens. Ces standards sont développés selon les conditions climatiques, culturelles et même politiques Européennes qui ne sont pas toujours adaptées pour l'Afrique. Toujours selon l'OMS, 70\% d'équipement médical venant des nations les plus développées ne fonctionne pas dans les hôpitaux des pays en voie de développement (P. HEIMANN, 2000).

Des études menées dans le cadre du programme de l'OMS montrent que dans trente-trois (33) hôpitaux de dix (10) pays en voie de développement, près d'un tiers des pannes d'équipements médicaux sont causées par l'instabilité des réseaux électriques. Tout ceci fait ressortir la problématique de l'inadéquation de ces appareils aux contextes des pays en développement (World Health Organization , 2010) (J. F. ATEBA, 2017). Pourtant, la déclaration d'Alma-Ata en 1978 (Organisation Mondiale de la Santé, 2006) de l'OMS a insisté sur l'importance de la technologie médicale appropriée, dans la délivrance de soins de santé qui a été reprise dans le projet DMP. Du fait de cette inadéquation, des résultats erronés, donnés par un dispositif 
médical peuvent être à l'origine d'un mauvais diagnostic et par conséquent mettre en danger la vie des patients ou du personnel médical. De même, cette inadéquation est à l'origine de la durée de vie réduite de ces équipements importés dont $96 \%$ ne sont plus fonctionnels après cinq ans de services (MOHR, 1987).

Certes, plusieurs travaux de recherches (Bretagne Commerce International, 2019) ont désigné l'instabilité du réseau électrique comme sources de pannes de ces appareils dans les pays en voie de développement, mais n'ont pas déterminé avec précision les perturbations (creux de tension, baisse de tension, coupure d'énergie, etc.) qui seraient à l'origine de cette situation.

Des informations recueillies auprès des ingénieurs biomédicaux des services de maintenance, de plusieurs hôpitaux au Cameroun montrent que plusieurs pannes liées à la destruction des générateurs des appareils de radiologie ont eu lieu pendant une coupure d'énergie électrique. Une coupure pouvant être définie comme une chute de tension supérieure à $90 \%$ de la valeur nominale, d'une durée inférieure à une minute si elle est brève ou supérieure à une minute si elle est longue (Philippe, 2001) (IGNATOVA, 2006).

En effet, les professionnels de la santé font souvent d'utiles suggestions pour améliorer les dispositifs médicaux (MALKIN, 2007). Malheureusement, le principal obstacle auquel ils se heurtent est d'amener leurs idées aux stades de la conception, de l'essai et de la fabrication (Organisation Mondiale de la Santé, 2020). Pourtant les pays émergeants constituent un marché important pour les grands fabricants mondiaux de dispositifs médicaux.

L’objectif du présent travail est de mener une étude scientifique, sur le fonctionnement des appareils de radiologie à haute fréquence sur le réseau Camerounais. Il sera précisément question d'étudier le comportement de ces appareils lors d'une coupure d'énergie électrique, pendant la phase de production des rayons $\mathrm{X}$ afin de comprendre les raisons de ces pannes régulières décriées par les professionnels de santé. Pour répondre à cet objectif, la première section de cet article décrit le matériel d'expérimentation et les méthodes utilisés. La seconde section est consacrée à la présentation des résultats de mesures effectuées. Et la dernière section est dédiée aux discussions.

\section{Matériel et Méthodes \\ Matériel}

Dans le cadre de ce travail, ont été utilisé le matériel suivant :

- un analyseur de réseau, le PQ Box 100 de A-Eberle, qui a servi à enregistrer les différentes perturbations, et son logiciel d'analyse de données WinPQ qui a permis d'analyser les enregistrements ; 
- ordinateur portable ;

- fiches d'enquêtes ;

- $\quad$ kVp mètre de marque DIAVOLT, propriété de l'Agence Nationale de Radioprotection (ANRP) qui a permis de mesurer les tensions d'alimentation des tubes afin d'observer leur évolution.

- et des appareils de radiologie qui ont fait l'objet de cette étude.

\section{Méthodes}

Cette étude s'est effectuée dans une dizaine d'hôpitaux Camerounais issus de quatre régions du pays (Centre, Sud, Ouest et Littoral), sur onze appareils de radiologie fonctionnant à haute fréquence entre Août 2018 et Août 2019 puis entre Décembre 2020 et Janvier 2021.

Le synoptique d'ensemble de la chaine de puissance d'un appareil de radiologie est donné à la Figure 1. L'analyse de cette figure permettra d'étudier et d'interpréter le comportement de l'appareil face aux perturbations électriques du réseau, à partir de l'alimentation jusqu'à la haute tension $(\mathrm{kV})$ appliquée aux bornes du tube à rayons $\mathrm{X}$. Cette analyse permettra aussi de circonscrire les termes essentiels à cette étude. En effet, l'objectif de ces appareils étant de produire les rayons X qui serviront à donner une image d'un organe particulier du patient, étudier ces équipements revient à étudier le cycle de production de ces rayonnements.

De manière générale, le fonctionnement du circuit d'alimentation du tube est le suivant :

L’alimentation est faite par le réseau triphasé BT d'ENEO (Entreprise qui gère la distribution de l'énergie électrique). Cette tension du réseau est redressée par un pont de 6 diodes (PD3) puis filtrée afin de réduire l'ondulation de la tension redressée. La tension redressée et filtrée, alimente alors un onduleur résonant, dont le rôle est de générer à sa sortie un courant alternatif de haute fréquence. Ce courant alternatif de haute fréquence permet d'alimenter le primaire d'un transformateur HT/HF. Le courant secondaire est redressé et filtré. Enfin, la tension ainsi obtenu est appliquée aux bornes du tube ce qui lui permettra de produire les rayons $\mathrm{X}$ pour irradier le patient. 


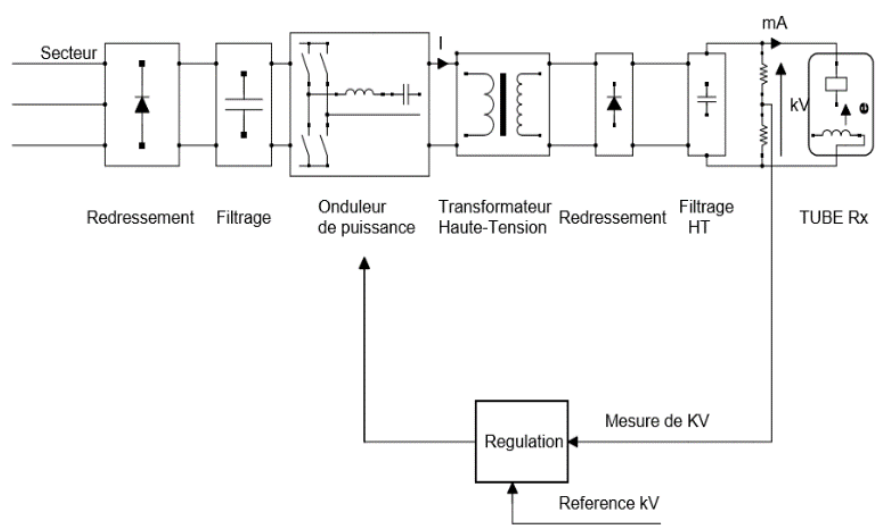

Figure 1. Synoptique d'ensemble de la chaine de puissance d'un appareil de radiologie à rayons $x$ à HF (LAEUFFER, 1997)

Le principe de fonctionnement des générateurs à haute fréquence est basé sur la relation entre la tension du secondaire $\left(\mathrm{U}_{2}\right)$ et la fréquence $(f)$ à l'entrée du transformateur, à savoir la formule de Boucherot (L. ALLAY, 1993):

$$
U_{2}=4,44 N_{2} \hat{B} S f
$$

$\mathrm{N}_{2}$ étant le nombre de spires du bobinage secondaire, B le champ magnétique maximal et $\mathrm{S}$ la section du circuit magnétique.

Cette relation de proportionnalité permet de comprendre que la variation de la fréquence à la sortie de l'onduleur entraine la variation de la tension au secondaire du transformateur et donc de la tension d'alimentation du tube à rayons $\mathrm{X}$.

\section{Tension d'alimentation}

La tension BT livrée par ENEO aux consommateurs Camerounais est constituée de trois tensions simples (phase-neutre) (Ministère de l'Eau et de l’Energie-Cameroun, 2009), régies par les équations ci-dessous :

$$
\begin{aligned}
& v_{1}(t)=V_{M} \sin (2 \pi f t) \\
& v_{2}(t)=v_{M} \sin \left(2 \pi f t+\frac{2 \pi}{3}\right) \\
& v_{3}(t)=v_{M} \sin \left(2 \pi f t+\frac{4 \pi}{3}\right)
\end{aligned}
$$

$f=50 \mathrm{~Hz}$ étant la fréquence du réseau, $\mathrm{V}_{\text {eff }}=220 \mathrm{~V}$ étant la valeur efficace de la tension et $\mathrm{V}_{\mathrm{M}}$ la valeur maximale de la tension en $\mathrm{V}$;

$$
V_{M}=V_{\text {eff }} \sqrt{2}
$$


Tableau 1. Valeurs normalisées des tensions efficaces du réseau (Ministère de l’Eau et de l'Energie-Cameroun, 2009)

\begin{tabular}{|c|c|c|}
\hline Tensions efficaces & Valeurs en volt & Précision \\
\hline Simples $\left(V_{\text {eff }}\right)$ & 220 & $\pm 10 \%$ \\
\hline Composées $\left(U_{e f f}\right)$ & 380 & $\pm 10 \%$ \\
\hline
\end{tabular}

Tableau 2. Valeurs normalisées des tensions maximales du réseau

\begin{tabular}{|c|c|c|}
\hline Tensions maximales $\left(V_{M}\right)$ & Valeurs en volt & Précision \\
\hline Simples & 311 & $\pm 10 \%$ \\
\hline
\end{tabular}

\section{Tension redressée (PD3)}

Pour un PD3, redressement à 6 pulsations, les grandeurs caractéristiques de la tension redressée, c'est-à-dire les tensions moyenne ( $\left.V_{c . m o y}\right)$ et efficace $\left(V_{c . \text { eff }}\right)$ sont respectivement (ZELLOUMA, 2006):

$$
\begin{aligned}
V_{c . \text { moy }} & =\frac{1}{\frac{T}{6} \int_{0}^{\frac{T}{6}} V_{c}(t) d t=\frac{3 \sqrt{3}}{\pi} V_{M}=\frac{3 \sqrt{3} \sqrt{2}}{\pi} V_{\text {eff }}} \\
V_{c . \text { eff }} & =\sqrt{\frac{1}{\frac{1}{6} \int_{0}^{\frac{T}{6}} V_{c}^{2}(t) d t}=\sqrt{3} V_{\text {eff }} \sqrt{\left(1+\frac{3 \sqrt{3}}{2 \pi}\right)}}
\end{aligned}
$$

\section{Onduleur résonant}

Les onduleurs à résonance sont apparus principalement grâce à la nécessiter de développer des alimentations hautes fréquences pour :

- les systèmes de chauffage par induction ;

- les générateurs de rayons $X$.

Il existe trois grandes familles des onduleurs à résonance (HELAIMI, 2014):

- Les onduleurs en pont en $\mathrm{H}$;

- les onduleurs en demi-pont;

- les onduleurs à deux circuits oscillants;

L’onduleur en pont en $\mathrm{H}$ est celui utilisé dans le cas étudié. En fonction de la disposition des composants de résonance, on peut citer : 


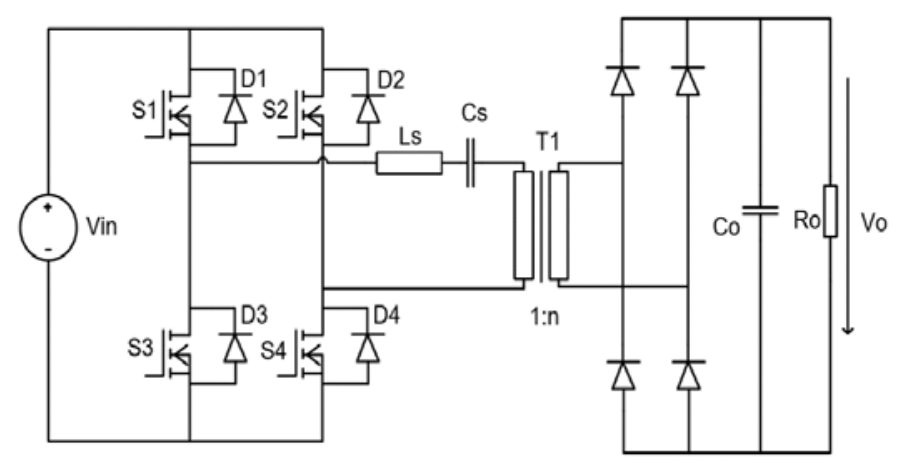

Figure 2. Onduleur série (CAVALCANTE, 2006)

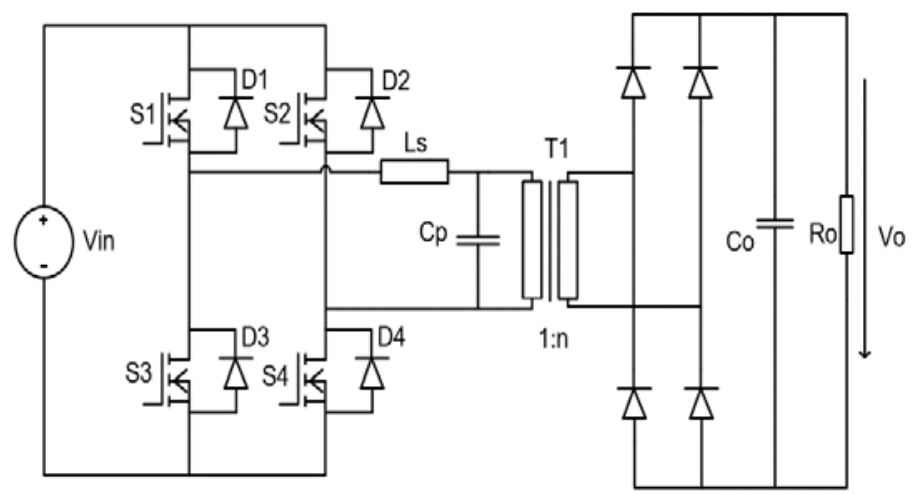

Figure 3. Onduleur parallèle (CAVALCANTE, 2006)

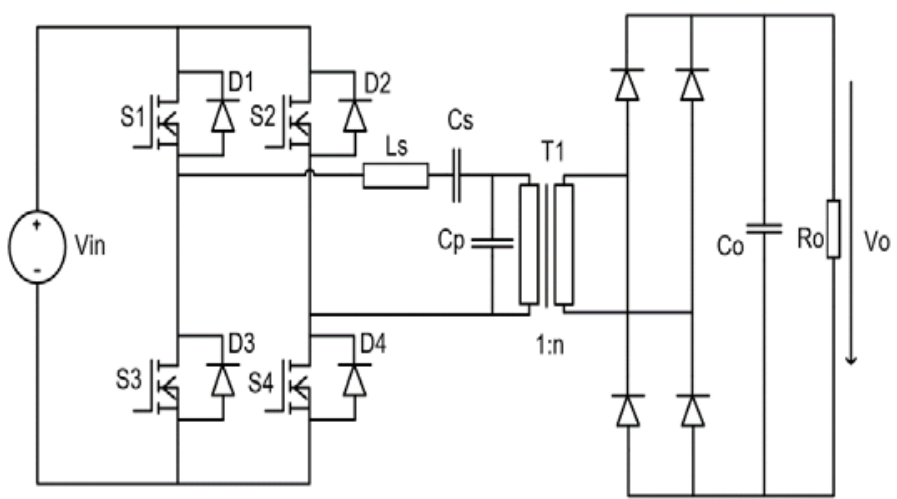

Figure 4. Onduleurs série-parallèle (CAVALCANTE, 2006)

Dans le domaine médical, plusieurs topologies d'onduleurs à résonance ont été développées ces dernières années en s'appuyant sur ces trois types ci-dessus cités, dans l'objectif de rendre les générateurs encore plus performants. Peuvent ainsi être cités : les onduleurs résonants LCLC, les 
onduleurs séries- parallèles LCC et les onduleurs multiniveaux (ZELLOUMA, 2006) (CAVALCANTE, 2006)

Dans le but de simplifier cette étude, et de bien circonscrire des notions essentielles à ce travail, la réflexion a été menée dans cette partie au tour de l’onduleur série.

La Figure 5 ci-dessous représente le schéma simplifié du système en tenant compte de la source alternative déjà redressée.

Circuit résonant: $(\mathrm{Lr}, \mathrm{Cr})$;

$f c=$ fréquence de commutation des interrupteurs IGBT ;

$f r=$ fréquence propre du circuit résonant ;

$Z \bar{Z}$ impédance du circuit résonant

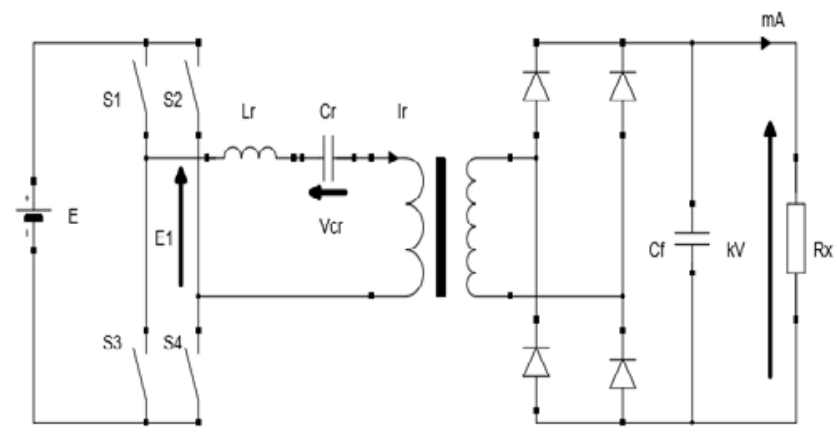

Figure 5. Schéma d’un convertisseur à résonance série

Ces convertisseurs résonants peuvent fonctionner en :

- hyper-résonance : $\boldsymbol{f}_{c}>\boldsymbol{f}_{\boldsymbol{r}}$

La source $E_{1}$ voit une charge globalement inductive, et est en avance de phase sur le courant $i_{r}$ (LAEUFFER, 1997). Le caractère sélectif du filtre LC autour de $\mathrm{f}_{\mathrm{r}}$ fait que $\mathrm{i}_{\mathrm{r}}$ soit quasiment sinusoïdal (FOREST, 1999).

Soient :

$v_{e}=E_{1}=$ tension de sortie de l'onduleur ;

$E=V_{\text {Ceff }}=$ valeur efficace de la tension redressée en BT ;

$i_{e}=i_{r}=$ courant dans le circuit oscillant $L_{r} C_{r}$;

$v_{c r}=$ tension aux bornes du condensateur ;

$v_{r}=$ tension aux bornes de l'ensemble $L_{r} C_{r}$;

$v_{s}=$ tension aux bornes du tube à rayon $\mathrm{x}$;

$i_{r}=$ courant dans le circuit oscillant ;

$V_{e f f}=$ tension efficace du réseau.

En considérant l'origine des temps identique au début de chaque phase (FOREST, 1999): 


$$
i_{e}=I \sin \left(\omega_{r} t+\phi\right)
$$

$I$ étant la valeur maximale du courant $i_{e}, \omega_{r}$ la pulsation du circuit résonant et $\phi$ la phase à l'origine.

$$
\omega_{r}=2 \pi f_{r}=\frac{1}{\sqrt{L_{r} C_{r}}}
$$

On démontre que (FOREST, 1999):

$V_{S}=m E \frac{-Q(1+\operatorname{Cos} \theta)+\sqrt{2 Q^{2}(1+\operatorname{Cos} \theta)+\left(1-\operatorname{Cos}^{2} \theta\right)^{2}}}{(1-\operatorname{Cos} \theta)+Q^{2}(1+\operatorname{Cos} \theta)}$

Avec

$\theta=\pi \frac{f_{r}}{f_{c}}$

et

$$
Q=\frac{\pi m^{2}}{2 R_{X}} \sqrt{\frac{L_{r}}{C_{r}}} \cdot \frac{f_{r}}{f_{c}}
$$

En remplaçant E par son expression de l'équation (7), on obtient :

$$
V_{S}=m \sqrt{3} \cdot V_{\text {eff }} \sqrt{\left(1+\frac{3 \sqrt{3}}{2 \pi}\right)} \frac{-Q(1+\cos \theta)+\sqrt{2 Q^{2}(1+\operatorname{Cos} \theta)+\left(1-\operatorname{Cos}^{2} \theta\right)^{2}}}{(1-\operatorname{Cos} \theta)+Q^{2}(1+\operatorname{Cos} \theta)}
$$

Hypo- résonance : $f_{c}<f_{r}$

La Source $E_{1}$ voit une charge globalement capacitive, la tension $E$ est en retard de phase sur le courant $I r$ (LAEUFFER, 1997).

En faisant le même raisonnement que précédemment, on a (FOREST, 1999) :

$V_{S}=m E \frac{Q(1+\operatorname{Cos} \theta)+\sqrt{2 Q^{2}(1+\operatorname{Cos} \theta)+(1-\operatorname{Cos} \theta)^{2}}}{(1-\operatorname{Cos} \theta)+Q^{2}(1+\operatorname{Cos} \theta)}$

En remplaçant E par son expression de l'équation (7), on obtient :

$V_{S}=m \sqrt{3} \cdot V_{\text {eff }} \sqrt{\left(1+\frac{3 \sqrt{3}}{2 \pi}\right)} \frac{Q(1+\operatorname{Cos} \theta)+\sqrt{2 Q^{2}(1+\operatorname{Cos} \theta)+(1-\operatorname{Cos} \theta)^{2}}}{(1-\operatorname{Cos} \theta)+Q^{2}(1+\operatorname{Cos} \theta)}$

La tension de sortie, dans les deux cas est proportionnelle à la tension efficace du réseau.

Elle est aussi fonction du rapport de transformation $m$ du transformateur HT/HF, des fréquences de résonance et de commutation des interrupteurs et enfin de la charge à alimenter.

Donc, toute variation de la valeur efficace ou maximale de la tension du réseau, pourrait modifier la tension aux bornes du tube à rayons $\mathrm{X}$. C'est la 
raison pour laquelle le système de régulation des $\mathrm{HT}$, qui est comme son nom l'indique, chargé de réguler la tension de sortie en fonction des consignes y est monté.

Cependant, en dépit de la présence des systèmes de régulation HT, les experts Camerounais reconnaissent à l'unanimité l'influence des coupures et des baisses de tension sur le fonctionnement des appareils de radiologie à HF. Dans le cadre de ce travail, seul le cas des coupures a été abordé.

Le Tableau 3 ci-dessous donne les valeurs des précisions normalisées des tensions $(\mathrm{kV})$ d'alimentation des tubes à rayons $\mathrm{X}$ utilisées par l'ANRP.

Tableau 3. Précisions normalisées des tensions $\mathrm{kV}$ utilisées lors des séances de contrôle qualité des appareils de radiologie conventionnelle (NJIKI Calvin Didier, 2018)

\begin{tabular}{|c|c|c|c|}
\hline Paramètre & bon & normal & mauvais \\
\hline Précisions des tensions $(\mathrm{kV})$ & $\leq 5 \%$ & $\leq 10 \%$ & $>10 \%$ \\
\hline
\end{tabular}

La méthodologie mise en œuvre se décline en trois points :

- une enquête portant sur l'effet des coupures d'énergie électrique sur les appareils de radiologie à rayons $\mathrm{X}$, auprès du personnel de santé ;

- des mesures des grandeurs électriques (courants, tensions, etc.) entrant dans la production des rayons $\mathrm{X}$;

- l'étude/analyse des données recueillies.

Etant donné l'importance et le coût élevé de ces appareils, aucun hôpital n'a accepté une quelconque simulation volontaire d'une coupure. Pour cette raison, cette étude s'est faite dans les conditions de fonctionnements habituels des appareils étudiés.

\section{Résultats}

Le résultat des enquêtes menées auprès des professionnels des services de radiologie et de maintenance est récapitulé dans le Tableau 4.

En effet, en dehors de quelques nouvelles recrues qui n'ont pas pris position, à l'unanimité tout le personnel des hôpitaux Camerounais où l'enquête a été effectuée a reconnu que les pannes des appareils de radiologie de ces dernières années ont été en grande partie causées par les coupures d'énergie électrique.

Tableau 4. Résultat de l'enquête menée auprès du personnel médical des hôpitaux où s’est déroulée cette étude

\begin{tabular}{|c|c|c|c|}
\hline Services & \multirow{2}{*}{$\begin{array}{c}\text { Personnel ayant } \\
\text { participé à } \\
\text { l'enquête }\end{array}$} & \multicolumn{2}{|c|}{$\begin{array}{c}\text { Personnel ayant reconnu des pannes } \\
\text { causées par des coupures }\end{array}$} \\
\cline { 3 - 4 } & 24 & Effectif & Pourcentage \\
\hline $\begin{array}{c}\text { Maintenance } \\
\text { biomédicale }\end{array}$ & & 23 & $95,8 \%$ \\
\hline $\begin{array}{c}\text { Service de } \\
\text { radiologie }\end{array}$ & 68 & 66 & $97 \%$ \\
\hline
\end{tabular}


Le Tableau 5 donne les valeurs, les précisions et les observations des mesures faites avec le $\mathrm{kVp}$ mètre. Les valeurs des tensions fixées sur le pupitre de commande sont celles qui sont les plus utilisées lors de la réalisation des images. Le nombre d'essai effectué par appareil dans le Tableau 5 est fixé par les experts de l'ANRP dans le cadre des contrôles qu'ils effectuent selon le protocole officiel de l'Agence. Le kVp mètre, de marque Diavolt, utilise les énergies des rayonnements émis pour déterminer la valeur de la tension $(\mathrm{kV})$ qui a permis leur production.

Nous observons au Tableau 5 que trois (03) essais ont fourni des tensions en dehors de la marge de 10\% prévu au Tableau 3 :

- appareil 2, essai $3: 18,7 \%$

- appareil 3, essai $4: 16,1 \%$

- $\quad$ appareil 9, essai $4: 12,2 \%$.

Ces dépassements nous informent de l'apparition des surtensions pendant ces essais respectifs. De plus le Tableau 5 fait état des coupures d'énergie à ces instants.

En somme, il y a eu apparition des surtensions pendant les coupures, ce qui n'est pas le cas pour les essais qui se sont terminés sans aucun incident.

Tableau 5. Mesures effectuées avec le kVp mètre

\begin{tabular}{|c|c|c|c|c|c|}
\hline & $\begin{array}{c}\text { Tension } \\
\text { fixée sur } \\
\text { le } \\
\text { pupitre } \\
(\mathrm{kV})\end{array}$ & $\begin{array}{c}\text { Tension } \\
\text { maximale } \\
\text { mesurée } \\
(\mathrm{kV})\end{array}$ & $\begin{array}{l}\text { Précision des } \\
\text { tensions } \\
\text { maximales } \\
\text { mesurées }\end{array}$ & $\begin{array}{c}\text { Coupure } \\
\text { pendant la } \\
\text { mesure. }\end{array}$ & Observation \\
\hline \multirow{5}{*}{ Appareil 1} & \multirow{5}{*}{70} & 71,0 & $1,43 \%$ & Non & Bon \\
\hline & & 70,7 & $1 \%$ & Non & Bon \\
\hline & & 71,2 & $1,7 \%$ & Non & Bon \\
\hline & & 70,0 & $0 \%$ & Non & Bon \\
\hline & & 71,0 & $1,4 \%$ & Non & Bon \\
\hline \multirow{8}{*}{ Appareil 2} & \multirow{5}{*}{70} & 71,0 & $1,4 \%$ & Non & Bon \\
\hline & & 70,8 & $1,1 \%$ & Non & Bon \\
\hline & & 83,1 & $18,7 \%$ & Oui & Mauvais \\
\hline & & 72,0 & $2,9 \%$ & Non & Bon \\
\hline & & 71,1 & $1,6 \%$ & Non & Bon \\
\hline & \multirow{3}{*}{100} & 102 & $2 \%$ & Non & Bon \\
\hline & & 101,8 & $1,8 \%$ & Non & Bon \\
\hline & & 101,8 & $1,8 \%$ & Non & Bon \\
\hline \multirow{9}{*}{ Appareil 3} & \multirow{5}{*}{70} & 72,1 & $3 \%$ & Non & Bon \\
\hline & & 72,0 & $2,9 \%$ & Non & Bon \\
\hline & & 71,7 & $2,4 \%$ & Non & Bon \\
\hline & & 81,3 & $16,1 \%$ & Oui & Mauvais \\
\hline & & 70,9 & $1,3 \%$ & Non & Bon \\
\hline & \multirow{4}{*}{70} & 72,6 & 3,71 & Non & Bon \\
\hline & & 72,7 & 3,86 & Non & Bon \\
\hline & & 72,7 & 3,86 & Non & Bon \\
\hline & & 72,7 & 3,86 & Non & Bon \\
\hline
\end{tabular}




\begin{tabular}{|c|c|c|c|c|c|}
\hline \multirow{6}{*}{ Appareil 4} & & 72,8 & 4,00 & Non & Bon \\
\hline & \multirow{5}{*}{81} & 85,9 & $6 \%$ & Non & Bon \\
\hline & & 86 & $6,2 \%$ & Non & Bon \\
\hline & & 85,8 & $5,9 \%$ & Non & Bon \\
\hline & & 86 & $6,2 \%$ & Non & Bon \\
\hline & & 85,8 & $5,9 \%$ & Non & Bon \\
\hline \multirow{3}{*}{ Appareil 5} & \multirow{3}{*}{100} & 94,3 & 5,7 & Non & Bon \\
\hline & & 94,3 & 5,7 & Non & Bon \\
\hline & & 94,0 & 6,0 & Non & Bon \\
\hline \multirow{10}{*}{ Appareil 6} & \multirow{5}{*}{28} & 30,1 & $7,5 \%$ & Non & Bon \\
\hline & & 30,0 & $7,1 \%$ & Non & Bon \\
\hline & & 30,1 & $7,5 \%$ & Non & Bon \\
\hline & & 30,1 & $7,5 \%$ & Non & Bon \\
\hline & & 30,0 & $7,1 \%$ & Non & Bon \\
\hline & \multirow{5}{*}{30} & 32,2 & $7,3 \%$ & Non & Bon \\
\hline & & 32,2 & $7,3 \%$ & Non & Bon \\
\hline & & 32,2 & $7,3 \%$ & Non & Bon \\
\hline & & 32,2 & $7,3 \%$ & Non & Bon \\
\hline & & 32,2 & $7,3 \%$ & Non & Bon \\
\hline \multirow{9}{*}{ Appareil 7} & \multirow{3}{*}{60} & 59 & $-1,7 \%$ & Non & Bon \\
\hline & & 59 & $-1,7 \%$ & Non & Bon \\
\hline & & 59,1 & $-1,8 \%$ & Non & Bon \\
\hline & \multirow{3}{*}{80} & 78,3 & $-2,1 \%$ & Non & Bon \\
\hline & & 78,3 & $-2,1 \%$ & Non & Bon \\
\hline & & 78,3 & $-2,1 \%$ & Non & Bon \\
\hline & \multirow{3}{*}{100} & 94,3 & $-5,7 \%$ & Non & Bon \\
\hline & & 94,3 & $-5,7 \%$ & Non & Bon \\
\hline & & 94 & $-6 \%$ & Non & Bon \\
\hline \multirow{9}{*}{ Appareil 8} & \multirow{3}{*}{60} & 60,5 & $0,8 \%$ & Non & Bon \\
\hline & & 60,1 & $0,2 \%$ & Non & Bon \\
\hline & & 60,6 & $1 \%$ & Non & Bon \\
\hline & \multirow{3}{*}{80} & 81,8 & $2,2 \%$ & Non & Bon \\
\hline & & 81,0 & $1,2 \%$ & Non & Bon \\
\hline & & 82,3 & $2,8 \%$ & Non & Bon \\
\hline & \multirow{3}{*}{100} & 103,1 & $3,1 \%$ & Non & Bon \\
\hline & & 103,1 & $3,1 \%$ & Non & Bon \\
\hline & & 103,0 & $3 \%$ & Non & Bon \\
\hline \multirow{11}{*}{ Appareil 9} & \multirow{3}{*}{60} & 60,0 & $0 \%$ & Non & Bon \\
\hline & & 59,9 & $-0,2 \%$ & Non & Bon \\
\hline & & 60,0 & $0 \%$ & Non & Bon \\
\hline & \multirow{4}{*}{80} & 80,1 & $0,1 \%$ & Non & Bon \\
\hline & & 80,1 & $0,1 \%$ & Non & Bon \\
\hline & & 80,0 & $0 \%$ & Non & Bon \\
\hline & & 89,8 & $12,2 \%$ & Oui & Mauvais \\
\hline & \multirow{3}{*}{100} & 101,7 & $1,7 \%$ & Non & Bon \\
\hline & & 101,8 & $1,8 \%$ & Non & Bon \\
\hline & & 101,5 & $1,5 \%$ & Non & Bon \\
\hline & & 71,0 & 1,4 & Non & Bon \\
\hline
\end{tabular}




\begin{tabular}{|l|c|c|c|c|c|}
\hline \multirow{3}{*}{$\begin{array}{l}\text { Appareil } \\
10\end{array}$} & \multirow{2}{*}{70} & 71,2 & $1,7 \%$ & Non & Bon \\
\cline { 3 - 6 } & \multirow{3}{*}{102} & 71,2 & 1,7 & Non & Bon \\
\cline { 3 - 6 } & & 101,6 & $0,4 \%$ & Non & Bon \\
\cline { 3 - 6 } & & 101,6 & $0,4 \%$ & Non & Bon \\
\cline { 3 - 6 } & \multirow{3}{*}{$\begin{array}{l}\text { Appareil } \\
11\end{array}$} & 701,6 & $0,4 \%$ & Non & Bon \\
\cline { 3 - 6 } & \multirow{3}{*}{70} & 71,1 & $1,6 \%$ & Non & Bon \\
\cline { 3 - 6 } & \multirow{3}{*}{125} & 70,9 & $1,3 \%$ & Non & Bon \\
\cline { 3 - 6 } & & 71,0 & $1,4 \%$ & Non & Bon \\
\cline { 3 - 6 } & & 127,5 & $2 \%$ & Non & Bon \\
\cline { 3 - 6 } & & 127,5 & $2,8 \%$ & Non & Bon \\
\hline
\end{tabular}

Il a été décidé après ce constat d'utiliser pour la suite de l'étude un analyseur de réseau afin de s'assurer qu'il ne s'agissait pas des erreurs de mesure. Ce dernier permet quant à lui d'obtenir les informations sur l'évolution des grandeurs électriques (Courants, tensions, fréquences, puissances etc.) aux bornes des appareils étudiés en cas de perturbation électrique.

Le Tableau 6 présente les valeurs maximales des tensions (simples) d'alimentations des appareils de radiologie, enregistrées par l'analyseur de réseau pendant la production des rayons $\mathrm{X}$.

Le Tableau 6 montre qu'un essai de l'appareil 3 se retrouve hors de la marge de précision de 10\% prévue au Tableau 2, Avec des précisions de 74\% et $75,4 \%$ respectivement sur la deuxième et troisième phase. Ces dépassements nous informent de l'apparition d'une surtension pendant cet essai.

De plus le Tableau 6 souligne qu'il y a eu coupure pendant cet essai. Ce constat signifie que la coupure d'énergie est la cause de cette surtension. Par contre, aux autres essais où il n'y a pas eu de coupure, aucune surtension n’a été observée.

Tableau 6. Mesures effectuées avec l'analyseur de réseau

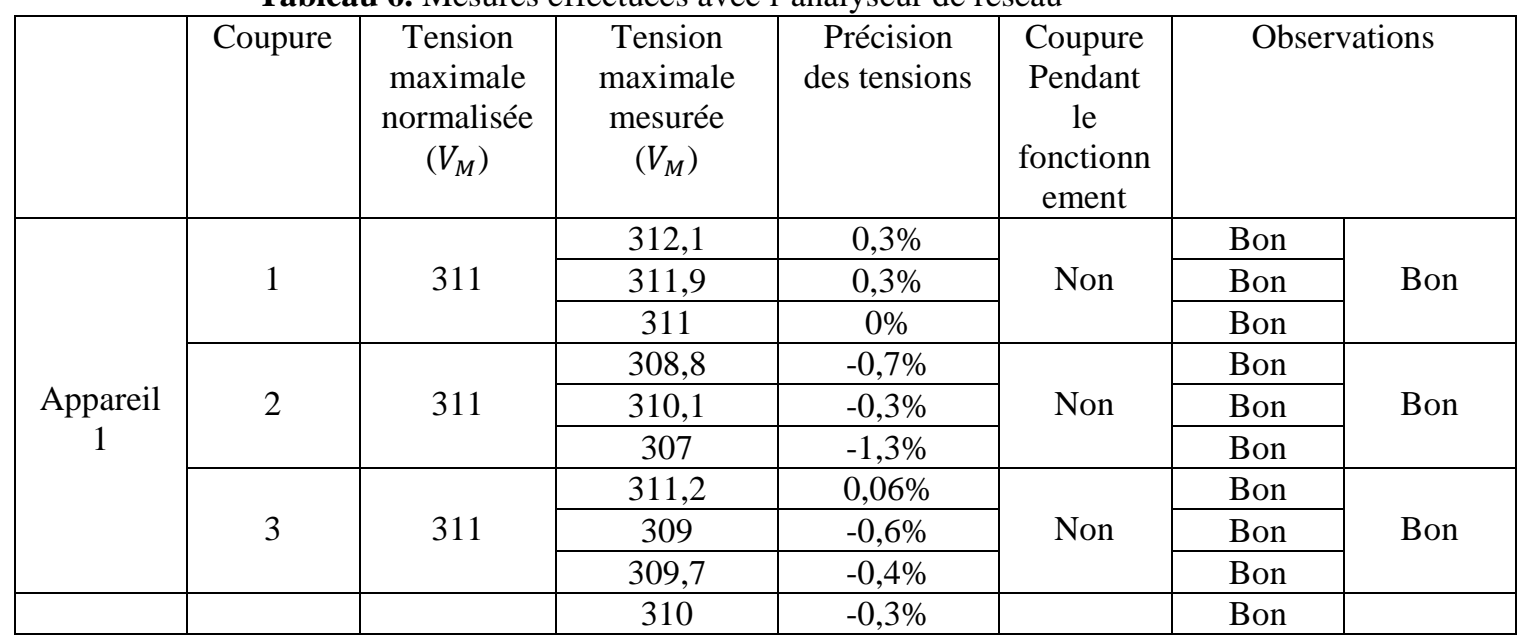




\begin{tabular}{|c|c|c|c|c|c|c|c|}
\hline \multirow{2}{*}{$\begin{array}{c}\text { Appareil } \\
2 \\
\end{array}$} & \multirow[t]{2}{*}{1} & \multirow[t]{2}{*}{311} & 308 & $-0,9 \%$ & \multirow[t]{2}{*}{ Non } & Bon & \multirow[t]{2}{*}{ Bon } \\
\hline & & & 308,9 & $-0,7 \%$ & & Bon & \\
\hline \multirow{15}{*}{$\begin{array}{c}\text { Appareil } \\
3\end{array}$} & \multirow{3}{*}{1} & \multirow{3}{*}{311} & 309,6 & $-0,4 \%$ & \multirow{3}{*}{ Oui } & Bon & \multirow{3}{*}{ Mauvais } \\
\hline & & & 540,4 & $74 \%$ & & Mauvais & \\
\hline & & & 545,6 & $75,4 \%$ & & Mauvais & \\
\hline & \multirow{3}{*}{2} & \multirow{3}{*}{311} & 309 & $-0,64 \%$ & \multirow{3}{*}{ Non } & Bon & \multirow{3}{*}{ Bon } \\
\hline & & & 310 & $-0,32 \%$ & & Bon & \\
\hline & & & 309,5 & $-0,48 \%$ & & Bon & \\
\hline & \multirow{3}{*}{3} & \multirow{3}{*}{311} & 314,6 & $1,15 \%$ & \multirow{3}{*}{ Non } & Bon & \multirow{3}{*}{ Bon } \\
\hline & & & 316,6 & $1,8 \%$ & & Bon & \\
\hline & & & 315,9 & $1,57 \%$ & & Bon & \\
\hline & \multirow{3}{*}{4} & \multirow{3}{*}{311} & 310 & $-0,3 \%$ & \multirow{3}{*}{ Non } & Bon & \multirow{3}{*}{ Bon } \\
\hline & & & 313,6 & $0,83 \%$ & & Bon & \\
\hline & & & 311,4 & $0,13 \%$ & & Bon & \\
\hline & & & 312,3 & $0,42 \%$ & & Bon & \\
\hline & 5 & 311 & 314,6 & $1,16 \%$ & Non & Bon & Bon \\
\hline & & & 312,4 & $0,45 \%$ & & Bon & \\
\hline & & & 309 & $-0,64 \%$ & & Bon & \\
\hline Appareil & 1 & 311 & 310,5 & $-0,2 \%$ & Non & Bon & Bon \\
\hline 4 & & & 309,9 & $-0.35 \%$ & & Bon & \\
\hline & & & 305,2 & $-1,8 \%$ & & Bon & \\
\hline & 1 & 311 & 307,2 & $-1,2 \%$ & Non & Bon & Bon \\
\hline & & & 306,7 & $-1,4 \%$ & & Bon & \\
\hline & & & 298,9 & $-3,8 \%$ & & Bon & \\
\hline & 2 & 311 & 302 & $-2,9 \%$ & Non & Bon & Bon \\
\hline & & & 300,7 & $-3,3 \%$ & & Bon & \\
\hline & & & 299,7 & $-3,6 \%$ & & Bon & \\
\hline & 3 & 311 & 302,8 & $-2,6 \%$ & Non & Bon & Bon \\
\hline & & & 301,7 & $-3 \%$ & & Bon & \\
\hline & & & 301,8 & $-3 \%$ & & Bon & \\
\hline & 4 & 311 & 318 & $2,2 \%$ & Non & Bon & Bon \\
\hline & & & 303,6 & $-2,4 \%$ & & Bon & \\
\hline & & & 300,2 & $-3,5 \%$ & & Bon & \\
\hline & 5 & 311 & 297,7 & $-4,3 \%$ & Non & Bon & Bon \\
\hline & & & 298,4 & $-4 \%$ & & Bon & \\
\hline Арparen & & & 281,6 & $-9,4 \%$ & & Bon & \\
\hline & 6 & 311 & 283,7 & $-8,7 \%$ & Non & Bon & Bon \\
\hline & & & 281,8 & $-9,4 \%$ & & Bon & \\
\hline & & & 283,3 & $-8,9 \%$ & & Bon & \\
\hline & 7 & 311 & 285,7 & $-8,1 \%$ & Non & Bon & Bon \\
\hline & & & 284,3 & $-8,6 \%$ & & Bon & \\
\hline & & & 305,1 & $-2 \%$ & & Bon & \\
\hline & 8 & 311 & 308 & $-1 \%$ & Non & Bon & Bon \\
\hline & & & 305,4 & $-1,8 \%$ & & Bon & \\
\hline & & & 307,8 & $-1 \%$ & & Bon & \\
\hline & 9 & 311 & 314,4 & $-1,1 \%$ & Non & Bon & Bon \\
\hline & & & 309,8 & $-0,4 \%$ & & Bon & \\
\hline
\end{tabular}




\begin{tabular}{|c|c|c|c|c|c|c|c|}
\hline & \multirow{3}{*}{10} & \multirow{3}{*}{311} & 304,7 & $-2 \%$ & \multirow{3}{*}{ Non } & Bon & \multirow{3}{*}{ Bon } \\
\hline & & & 307,8 & $-1 \%$ & & Bon & \\
\hline & & & 309 & $-0,6 \%$ & & Bon & \\
\hline & \multirow{3}{*}{11} & \multirow{3}{*}{311} & 302,3 & $-2,8 \%$ & \multirow{3}{*}{ Non } & Bon & \multirow{3}{*}{ Bor } \\
\hline & & & 304,2 & $-2,2 \%$ & & Bon & \\
\hline & & & 301,7 & $-3 \%$ & & Bon & \\
\hline & \multirow{3}{*}{12} & \multirow{3}{*}{311} & 302,8 & $-2,6 \%$ & \multirow{3}{*}{ Non } & Bon & \multirow{3}{*}{ Bor } \\
\hline & & & 305 & $-2 \%$ & & Bon & \\
\hline & & & 303,3 & $-2,5 \%$ & & Bon & \\
\hline \multirow{30}{*}{$\begin{array}{c}\text { Appareil } \\
6\end{array}$} & \multirow{3}{*}{1} & \multirow{3}{*}{311} & 281,5 & $-9,5 \%$ & \multirow{3}{*}{ Bon } & Bon & \multirow{3}{*}{ Bor } \\
\hline & & & 283,5 & $-8,8 \%$ & & Bon & \\
\hline & & & 284,6 & $-8,5 \%$ & & Bon & \\
\hline & \multirow{3}{*}{2} & \multirow{3}{*}{311} & 281 & $-9,6 \%$ & \multirow{3}{*}{ Non } & Bon & \multirow{3}{*}{ Bor } \\
\hline & & & 281,3 & $-9,5 \%$ & & Bon & \\
\hline & & & 282,8 & $-9 \%$ & & Bon & \\
\hline & & & 307 & $-1,2 \%$ & & Bon & \\
\hline & 3 & 311 & 293,4 & $-5,6 \%$ & Non & Bon & Bon \\
\hline & & & 295 & $-5,1 \%$ & & Bon & \\
\hline & & & 300 & $-3,5 \%$ & & Bon & \\
\hline & 4 & 311 & 303,6 & $-2,3 \%$ & Non & Bon & Bon \\
\hline & & & 301,7 & $-3 \%$ & & Bon & \\
\hline & & & 302,2 & $-2,82 \%$ & & Bon & \\
\hline & 5 & 311 & 305,5 & $-1,7 \%$ & Non & Bon & Bon \\
\hline & & & 303,1 & $-2,5 \%$ & & Bon & \\
\hline & & & 300 & $-3,5 \%$ & & Bon & \\
\hline & 6 & 311 & 298,1 & $-4,1 \%$ & Non & Bon & Bon \\
\hline & & & 300,8 & $-3,2 \%$ & & Bon & \\
\hline & & & 300,6 & $-3,3 \%$ & & Bon & \\
\hline & 7 & 311 & 303,5 & $-2,4 \%$ & Non & Bon & Bon \\
\hline & & & 302,2 & $-2,8 \%$ & & Bon & \\
\hline & & & 285,2 & $-8,3 \%$ & & Bon & \\
\hline & 8 & 311 & 288 & $-7,4 \%$ & Non & Bon & Bon \\
\hline & & & 297,5 & $-4,3 \%$ & & Bon & \\
\hline & & & 302 & $-2,9 \%$ & & Bon & \\
\hline & 9 & 311 & 303,6 & $-2,4 \%$ & Non & Bon & Bon \\
\hline & & & 302,2 & $-2,8 \%$ & & Bon & \\
\hline & & & 298,4 & $-4 \%$ & & Bon & \\
\hline & 10 & 311 & 302,3 & $-2,8 \%$ & Non & Bon & Bon \\
\hline & & & 300,5 & $-3,4 \%$ & & Bon & \\
\hline & & & 314,2 & $1 \%$ & & Bon & \\
\hline & 1 & 311 & 317,3 & $2 \%$ & Non & Bon & Bon \\
\hline & & & 315,6 & $1,5 \%$ & & Bon & \\
\hline & & & 307,3 & $-1,2 \%$ & & Bon & \\
\hline Appareil & 2 & 311 & 307,5 & $-1,1 \%$ & Non & Bon & Bon \\
\hline 7 & & & 308,3 & $-0,9 \%$ & & Bon & \\
\hline & & & 300,1 & $-3,5 \%$ & & Bon & \\
\hline & 3 & 311 & 302 & $-2,9 \%$ & Non & Bon & Bon \\
\hline & & & 302,4 & $-2,8 \%$ & & Bon & \\
\hline
\end{tabular}


October 2021 edition Vol.17, No.37

\begin{tabular}{|c|c|c|c|c|c|c|c|}
\hline \multirow{6}{*}{$\begin{array}{c}\text { Appareil } \\
8\end{array}$} & \multirow{3}{*}{1} & \multirow{3}{*}{311} & 299,1 & $-3,8 \%$ & \multirow{3}{*}{ Non } & Bon & \multirow{3}{*}{ Bon } \\
\hline & & & 306 & $-1,6 \%$ & & Bon & \\
\hline & & & 308 & $-1 \%$ & & Bon & \\
\hline & \multirow{3}{*}{2} & \multirow{3}{*}{311} & 311,1 & $0,03 \%$ & & Bon & \multirow{3}{*}{ Bon } \\
\hline & & & 310 & $-0,3 \%$ & & Bon & \\
\hline & & & 312 & $0,3 \%$ & & Bon & \\
\hline \multirow{6}{*}{$\begin{array}{c}\text { Appareil } \\
9\end{array}$} & \multirow{3}{*}{1} & \multirow{3}{*}{311} & 313,7 & $0,9 \%$ & \multirow{3}{*}{ Non } & Bon & \multirow{3}{*}{ Bor } \\
\hline & & & 312,8 & $0,6 \%$ & & Bon & \\
\hline & & & 312,2 & $0,4 \%$ & & Bon & \\
\hline & \multirow{3}{*}{2} & \multirow{3}{*}{311} & 308,4 & $-0,8 \%$ & \multirow{3}{*}{ Non } & Bon & \multirow{3}{*}{ Bor } \\
\hline & & & 307 & $-1,3 \%$ & & Bon & \\
\hline & & & 309,1 & $-0,6 \%$ & & Bon & \\
\hline \multirow{6}{*}{$\begin{array}{c}\text { Appareil } \\
10\end{array}$} & \multirow{3}{*}{1} & \multirow{3}{*}{311} & 306 & $-1,6 \%$ & \multirow{3}{*}{ Non } & Bon & \multirow{3}{*}{ Bor } \\
\hline & & & 306,4 & $-1,5 \%$ & & Bon & \\
\hline & & & 308,5 & $-0,8 \%$ & & Bon & \\
\hline & \multirow{3}{*}{2} & \multirow{3}{*}{311} & 310,6 & $-0,1 \%$ & \multirow{3}{*}{ Non } & Bon & \multirow{3}{*}{ Bon } \\
\hline & & & 309,1 & $-0,6 \%$ & & Bon & \\
\hline & & & 309,5 & $-2,7 \%$ & & Bon & \\
\hline \multirow{18}{*}{$\begin{array}{c}\text { Appareil } \\
11\end{array}$} & & & 311,9 & $0,3 \%$ & & Bon & \\
\hline & 1 & 311 & 313,1 & $0,7 \%$ & Non & Bon & Bon \\
\hline & & & 310 & $-0,3 \%$ & & Bon & \\
\hline & & & 311 & $0 \%$ & & Bon & \\
\hline & 2 & 311 & 310,2 & $-0,2 \%$ & Non & Bon & Bon \\
\hline & & & 309 & $-0,6 \%$ & & Bon & \\
\hline & & & 294 & $-5,5 \%$ & & Bon & \\
\hline & 3 & 311 & 292,3 & $-6 \%$ & Non & Bon & Bon \\
\hline & & & 289 & $-7 \%$ & & Bon & \\
\hline & & & 306,1 & $-1,6 \%$ & & Bon & \\
\hline & 4 & 311 & 308,9 & $-0,7 \%$ & Non & Bon & Bon \\
\hline & & & 310 & $-0,3 \%$ & & Bon & \\
\hline & & & 312,6 & $0,5 \%$ & & Bon & \\
\hline & 5 & 311 & 313,1 & $0,7 \%$ & Non & Bon & Bon \\
\hline & & & 314,1 & $1 \%$ & & Bon & \\
\hline & & & 308,8 & $-0,7 \%$ & & Bon & \\
\hline & 6 & 311 & 310,3 & $-0,2 \%$ & Non & Bon & Bon \\
\hline & & & 309,5 & $-0,5 \%$ & & Bon & \\
\hline
\end{tabular}

En somme, il y a apparition des surtensions lors des coupures d'énergie ayant eu lieu pendant la production des rayons $\mathrm{X}$ cela se vérifie dans les deux cas. 


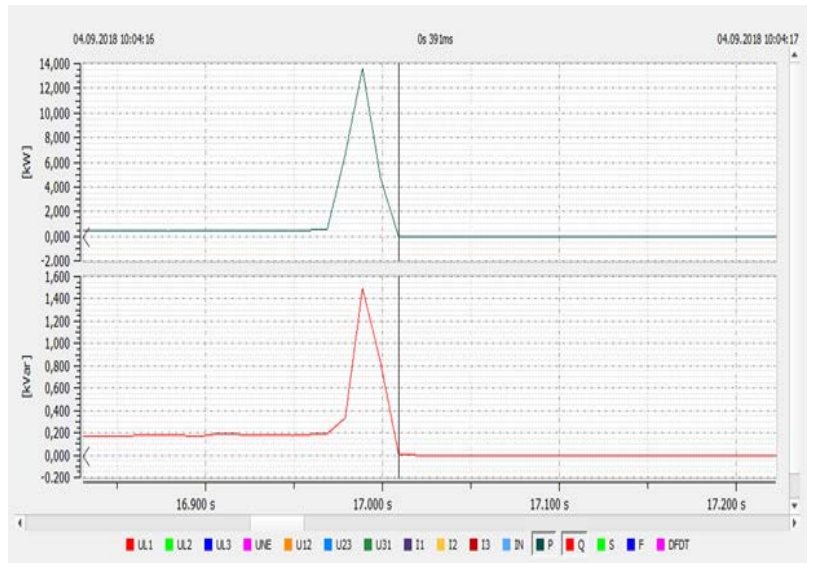

Figure 6. Evolution des puissances active et réactive lors de la coupure d'énergie pendant la phase de production des rayons $\mathrm{X}$

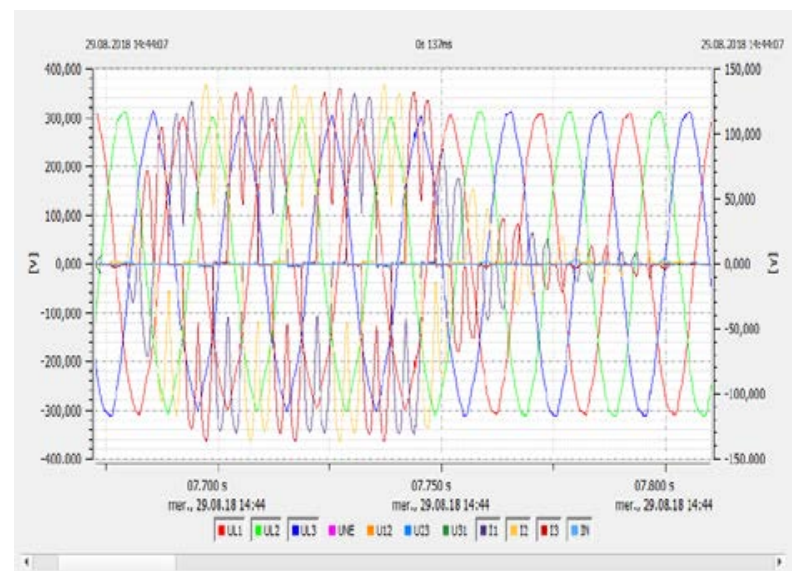

Figure 7. Evolution des courants et tensions instantanés pendant la phase de production des rayons $X$

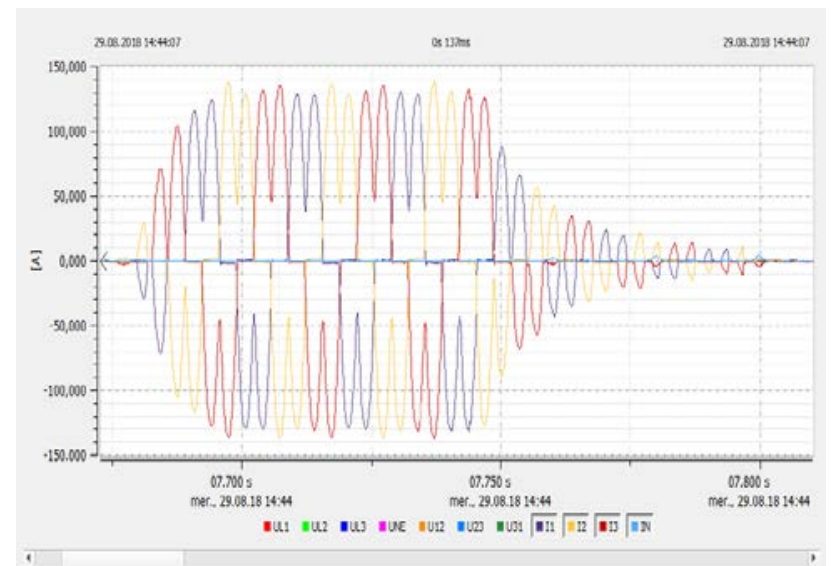

Figure 8. Evolution des courants pendant la phase de production des rayons $X$ 


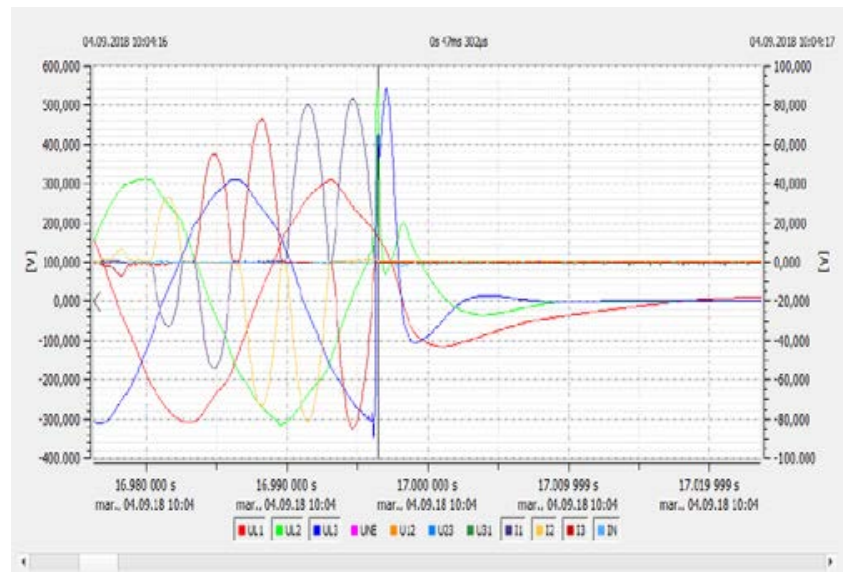

Figure 9. Evolution des courants et tensions instantanés lors d'une coupure d'énergie pendant la phase de production des rayons $X$ (appareil 3, essai 1)

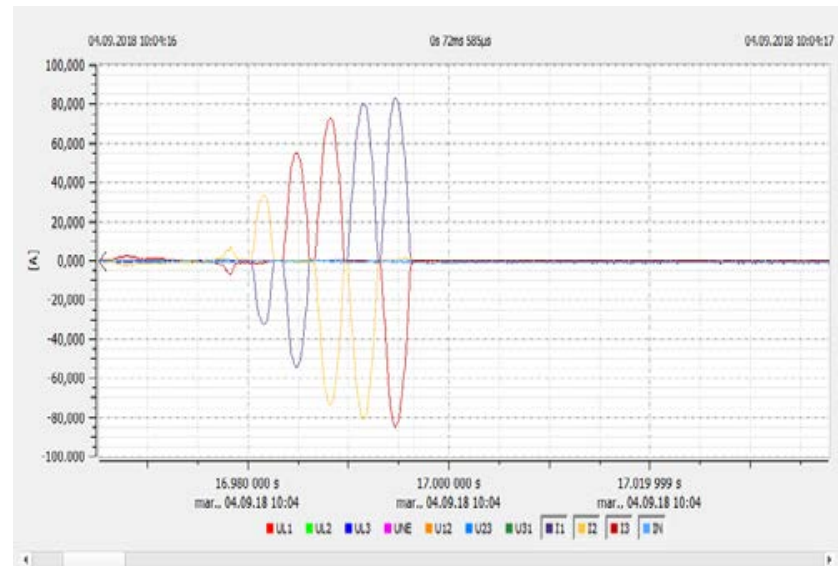

Figure 10. Evolution des courants lors de la coupure d'énergie pendant la phase de production des rayons $X$ : surtension (appareil 3, essai 1)

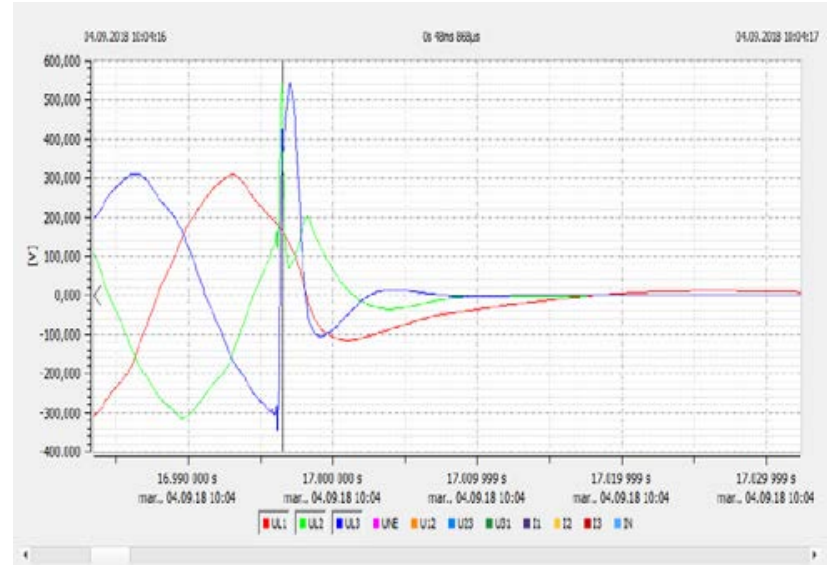

Figure 11. Evolution des tensions lors de la coupure d'énergie pendant la phase de production des rayons $X$ : surtension (appareil 3, essai 1, Tableau 6) 


\section{Discussion}

La scrutation de l'évolution de la puissance réactive sur la Figure 6 montre qu'à l'instant de l'enregistrement elle est positive. Cela signifie que la partie réactive de cet appareil est inductive et donc ne tolère pas de discontinuité du courant (AZAN.J, 1993). Cela signifie qu'il fonctionne en hyper-résonnance.

Les Figures $\mathbf{7}$ et $\boldsymbol{8}$ présentent respectivement les courbes des tensions et courants et, les courbes des courants des trois phases pendant le fonctionnement normal de cet appareil de radiologie.

L'atteinte des fréquences de commutation élevées, nécessite l'utilisation des montages dans lesquels tous les interrupteurs fonctionnent en commutation douce (G. SEGUIER, 2015). Il s'agit alors d'assurer une commutation (ouverture ou fermeture) commandée, fortement adoucie par passage du courant par zéro dans l'interrupteur (zero current switching, ZCS) ou par passage par zéro de la tension aux bornes de l'interrupteur (zero voltage switching, ZVS) (G. SEGUIER, 2015). Dans tous les cas, une commutation adoucie nécessite l'annulation de la tension et/ou du courant. Ces dispositifs (ou circuits) d'aide à la commutation permettent pendant le fonctionnement des convertisseurs, de réduire ou d'annuler les pertes à la commutation (G. SEGUIER, 2015) (Imed, 2020). Dans les cas étudiés, les coupures d'énergie se sont produites pendant le fonctionnement des appareils après l'enclenchement du processus de production des rayons X. Ces coupures étant des événements, l'analyseur de réseau a enregistré les Figures 6, 9, 10 et 11 représentent respectivement les allures des puissances consommées, des courants et tensions, des courants et enfin des tensions.

La lecture des Figures $\mathbf{9}$ et $\mathbf{1 1}$ montre qu'il y a apparition, des surtensions instantanées où les valeurs crêtes (maximales) des trois tensions simples sont $\mathrm{V}_{\mathrm{L} 1 \mathrm{M}}=309,6 \mathrm{~V}$ (bon), $\mathrm{V}_{\mathrm{L} 2 \mathrm{M}}=540,4 \mathrm{~V}$ (mauvais) et $\mathrm{V}_{\mathrm{L} 3 \mathrm{M}}=545,6 \mathrm{~V}$ (mauvais).

Les Figures $\mathbf{9}$ et $\mathbf{1 0}$ montrent à la fin du cycle que l'annulation des courants n'est pas progressive comme sur la Figure 7.

En effet une inductance se comporte comme un réservoir d'énergie électrique. Une charge inductive d'inductance L, parcouru par une intensité $i(t)$ accumule une énergie électromagnétique $w(t)$ (AZAN.J, 1993) donnée par :

$$
\begin{aligned}
& w(t)=\frac{1}{2} L i^{2}(t) \\
& w(t)=\frac{1}{2 L} \Phi^{2}
\end{aligned}
$$

$\Phi$ étant le flux magnétique. 


$$
\Phi=\operatorname{Li}(t)
$$

Cette énergie emmagasinée doit être évacuée de façon convenable avant la mise en arrêt de la dite charge. Il serait alors inadéquat de la libérer brutalement car, cela impliquerait une variation brutale (rapide) du flux magnétique synonyme d'apparition d'une surtension (Imed, 2020). Cette énergie étant proportionnelle au carré du flux magnétique comme l'indique l'Equation (17), sa libération brutale équivaut à une variation rapide de ce flux d'où l'apparition d'une surtension $e$ :

$$
e=-\frac{d \Phi}{d t}
$$

Cet inconvénient est généralement évité par l'utilisation d'un circuit d'aide à la commutation (CALC) abordé plus haut, qui assure le passage du courant si l'interrupteur correspondant est ouvert (Akpé, 1996).

Dans les cas présents, les appareils étant en fonctionnement, certains interrupteurs des onduleurs à résonance étaient fermés à l'instant des coupures. Les énergies emmagasinées se sont alors évacuées brutalement et, principalement à travers ces interrupteurs dès la disparition de la tension d'alimentation, ce qui n'a pas permis aux CALC de les éliminer convenablement. Les libérations brutales (rapides) de ces énergies dans les circuits en amont des charges ont donc engendré les variations rapides des flux magnétiques et donc des courant $i_{e}$ à la sortie des onduleurs. Ce qui explique l'apparition des surtensions qui ont été enregistrées par les appareils de mesure, illustrés aux figures $\mathbf{9}$ et $\mathbf{1 1}$.

Cette situation corrobore avec certains travaux menés sur les hacheurs alimentant des charges inductives qui attestent que, lorsqu'un dispositif d'alimentation s'éteint rapidement, l'énergie accumulée dans l'inductance est dissipée dans l'interrupteur statique cela provoque une surtension à travers le dispositif (Akpé, 1996). Ce qui explique le constat du personnel de santé sur les pannes des appareils de radiologie pendant les coupures.

Le flux étant proportionnel au courant comme indiqué à l'Equation (18), sa variation rapide est équivalente à une variation rapide du courant.

Le but des onduleurs à résonance est de permettre aux semiconducteurs de puissance de se placer à des positions plus favorables lors de leurs commutations en réduisant les $\frac{d i}{d t}$ et les $\frac{d v}{d t}$ (LAEUFFER, 1997). Cependant le présent travail a permis de comprendre que pour ces appareils triphasés à haute fréquence, équipés d'onduleurs à résonance, les coupures d'énergie restent un problème car celles-ci engendrent des surtensions qui peuvent détruire les composants, le tube à rayons $\mathrm{X}$ ou d'autres charges connectées au même point d'alimentation. Ces surtensions peuvent être aussi dangereuses pour le patient, le manipulateur et l'environnement car, elles peuvent engendrer des rayonnements de très hautes énergies, dangereux pour 
les êtres vivants (Stephanix radiological solutions, 2015). En somme, cette situation est très complexe car l'implémentation de la radioprotection demeure approximative en Afrique au sud du Sahara (Mbo Amvene J, 2017) (Kouandongui Bangue Songrou Francky, 2019) (P. Ongolo ZOGO, 2013).

De plus, comme mentionné plus haut, à l'exception de quelques nouvelles recrues, chaque personnel des services de radiologie et de maintenance rencontré pendant cette étude a au moins une fois déjà été témoin d'une panne d'appareil de radiologie à rayons $\mathrm{X}$ à haute fréquence survenue lors d'une coupure d'énergie électrique comme l'indique le résultat de l'enquête au Tableau 4.

En abordant le problème de coupure, nous nous sommes attaqués à une cause majeure des pannes des appareils de radiologie utilisés dans les pays en voie de développement où, l'instabilité du réseau électrique a toujours été indexée par les experts (MALKIN, Barriers for medical devices for the developing world, 2007) sans toutefois désigner une perturbation en particulier. Ce qui soulève la problématique de l'inadéquation de ces appareils et de leurs normes de conception et de fabrication aux contextes de leurs lieux d'utilisation dans les pays du Sud (Werner JF, 2010). En effet, ces appareils sont conçus et fabriqués dans les pays développés, selon leurs normes et, leurs contextes culturels et socio-économiques et où les coupures d'énergie électrique sont très rares. Le résultat de cette étude résonne comme une interpellation sur la nécessité de repenser le cadre normatif, afin de développer les appareils de radiologie à rayons $\mathrm{X}$ qui seront désormais en exploitation dans les pays en voie développement en général et au Cameroun en particulier, dans le but de les adapter aux réalités locales compte tenu de la régularité des coupures d'énergie électrique. Sur ce point, une des solutions efficaces serait de doter ces appareils d'une batterie et d'un onduleur incorporés à leur carte d'alimentation pendant leur fabrication. Ainsi, lors d'une éventuelle perturbation électrique ou d'une coupure, la batterie fournira l'énergie nécessaire pour terminer la séance de prise d'image et d'arrêter ensuite l'appareil sans aucun risque de surtension (Globaldiagnostix, 2021).

Une deuxième serait de recourir aux sources d'énergies renouvelables pour pouvoir utiliser ses appareils en mode hors réseau. Dans ce dernier cas, les appareils de radiologies auront leur source d'énergie propre fournie par un système solaire à base des panneaux.

\section{Conclusion}

Une étude des effets des coupures d'énergie électrique sur les appareils de radiologie à rayons $\mathrm{X}$ à haute fréquence a été conduite. Il était question tout d'abord de présenter l'appareil étudié, puis mener une étude théorique des différentes tensions permettant la production des rayons X. Ensuite, se basant sur des résultats d'enquêtes menées auprès des professionnels de santé, il a été 
démontré que les coupures d'énergie électrique sont les causes principales des pannes de ces appareils. Enfin, des mesures des grandeurs électriques aux bornes des appareils étudiés pendant leurs fonctionnements ont prouvé que lors des coupures ayant lieu pendant la production des rayons $\mathrm{X}$, ces appareils engendrent des surtensions lorsqu'ils fonctionnent en hyper résonance. Ce qui contribue non pas au rétablissement du patient mais plutôt à un aggravement de sa situation sanitaire à travers des maladies telles que des blessures, de destruction de cellules etc... Il est impératif que de dispositions soient prises au niveau des plateaux techniques sanitaires afin d'assurer la stabilité des tensions.

\section{References:}

1. Akpé, A. (1996). Modélisation et mise en eouvre de l'IGBT dans un onduleur de moyennepuissance. Lorraine: Institut National Polytechnique de Lorraine.

2. Amilcare. (2020, Octobre 10). Elettro Amici. Récupéré sur Site web Elettro Amici: http: //www.elettroamici.org/fr/

3. Azan.J. (1993). Tome 1: Précis d'électronique. Paris: Bréal.

4. Bretagne Commerce International. (2019, Decembre 20). Bretagne Commerce International. Récupéré sur Site web Bretagne Commerce International: https://www.bretagnecommerceinternational.com

5. Cavalcante, F. D. (2006). High output voltage series-Parallel resonant DC-DC converter for medical X-ray imaging application. Zurich: Swiss Federal Institute of Technology, Thesis of Ph.D.

6. Forest, J. P. (1999). Alimentation à découpage, Convertisseurs à résonance. Paris : Dunod.

7. G. Seguier, F. L. (2015). Electronique de puissance : structures, commandes, applications. Paris: Dunod.

8. Helaimi, M. (2014). Contribution à la commande d'un onduleur à résonance destiné au chauffage par induction. Oran: Université des Sciences et de la Technologie d'Oran. Thèse de Doctorat Ph.D.

9. Ignatova, V. (2006). Méthodes d'analyse de la qualité de l'énergie électrique. Application aux creux de tension et à la pollution harmonique. Grenoble: Université Joseph Fourrier. Thèse de Doctorat Ph.D.

10. Imed, H. (2020, Juin 22). Institut supérieur des études technologiques de Nabeul. Récupéré sur Site web Institut supérieur des études technologiques de Nabeul: http://www.isetn.rnu.tn

11. J. F. Ateba, A. S. (2017). Operational Measures Taken for the Authorization of X-ray Generators Used in the Medical Sector in Cameroon. Health Physics, 113(5) 414-418. 
12. Kouandongui Bangue Songrou Francky, B. T. (2019). Etat de la Radiologie Dans les Chu de Bangui et de Bimbo, Centrafrique. European Scientific Journal, 15(6): 1-9.

13. L. Allay, J. D. (1993). Physique Appliquée : Electronique Electrotechnique. Luçon: NATHAN.

14. Laeuffer, J. (1997). Convertisseur à résonance dans l'imagerie médicale. 3EI 1997- Journées.

15. Mainsah, B. O. (2008). Important factors for the design of medical devices for developing countries. Worcester: Faculty of the Worcester Polytechnic Institute.

16. Malkin, R. A. (2007). Barriers for medical devices for the developing worl. Expert Review of Medical Devices, 4(6): 759-763.

17. Malkin, R. A. (2007). Design of Health Care Technologies for the Developing World. Annual Review of Biomedical Engineering, 9(1): 567-587.

18. Mbo Amvene J, D. B. (2017). Observance des Règles de Radioprotection dans les Servicesd'Imagerie des Hôpitaux de l'Extrême-Nord du Cameroun. Health Sciences and Diseases,, 18(2): 83- 87.

19. Ministère de l'Eau et de l'Energie-Cameroun. (2009). Arrêté $\mathrm{N}^{\circ} 00000013 / \mathrm{MINEE}$ du 26 Janvier 2009 portant approbation du règlement du service de distribution publique d'électricité de la société AES-SONEL. Yaoundé : Ministère de l'Eau et de l'EnergieCameroun.

20. Mohr, J. P. (1987). Distributing and Transferring Medical Technology. International Journal of Technology Assessment and Health Care, 3(2): 281-291.

21. Njiki Calvin Didier, N. M. (2018). Quality Control of Conventional Radiology Devices in Selected Hospitals of the Republic Of Cameroon. International Journal of Innovative Sciences, Engineering and Technology, 5(3): 269-672.

22. Organisation Mondiale de la Santé. (2006). The Role of Medical Devices and Equipment in Contemporary Health Care Systems and Services. Geneva: World Health Organisation.

23. Organisation Mondiale de la Santé. (2020, Novembre 11). Organisation Mondiale de la Santé. Récupéré sur Site web Organisation Mondiale de la Santé: https://www.who.int

24. Organisation Mondiale dela Santé. (2012). Dispositifs médicaux : comment résoudre l'inadéquation ? Villars-sous-Yens: Organisation Mondiale de la Santé.

25. P. HEIMANN, A. I. (2000). Guidelines for health care equipment donations. Geneva: World Health Organisation. 
26. P. Ongolo Zogo, M. N. (2013). Connaissances en matière de radioprotection : enquête auprès des personnels des services hôspitaliers de radiodiagnostic, radiothérapie et médecine nucléaire a Yaoundé Cameroun. Radioprotectio, 48(1): 39-49.

27. Philippe, F. (2001). Cahier Technique $\mathrm{N}^{\circ}$ 199: La qualité de l'énergie électrique,. Schneider Electric.

28. Stephanix radiological solutions. (2015). HF series generators compact X-ray Unit. La Ricamarie: Stephanix radiological solutions.

29. Werner JF, D. I. (2010). L’imagerie par résonance magnétique haut champ en afrique subsaharienne une expérience sénégalaise. Journal Africain de l’Imagerie Médicale, 3(5): 222-247.

30. World Health Organization . (2010). Context dependency of medical devices. Geneva: World Health Organization.

31. World Health Organization. (2010). Guidelines for health care equipment donations. Geneva: World Health Organization.

32. Zellouma. (2006). Filtrage actif parallèle des harmoniques du courant genéré par un pont redresseur triphasé non commandé. Annaba: Université de Annaba-Badji Mokhtar, Ph.D thesis.

33. Globaldiagnostix. (2021, 01 Septembre). Globaldiagnostix. Récupéré sur le site : http://globaldiagnostix.org/ 